\title{
Issues in the safety testing of metabolites
}

\begin{abstract}
"The US FDA guideline on MIST provides considered practical guidance regarding what needs to be done to address potential safety concerns that may be posed by drug metabolites going forward. This guidance has already had a significant impact on the pharmaceutical industry..."
\end{abstract}

The new US FDA guidance on the safety testing of metabolites (MIST) provides considered criteria regarding when additional testing should be conducted in order to satisfy the regulator that the safety implications of drug metabolites have been thoroughly assessed. A number of other guidelines also touch on the subject of safety and metabolites. Here, the implications for drug development of this guidance on the safety of metabolites are placed into context, whilst an overview on current industrial approaches and strategies is also provided.

The production of metabolites as part of the disposition and elimination of drugs from the body has always been an area of active consideration for drug discovery and development. Thus, the presence of metabolites in the systemic circulation often triggers a range of questions concerning their potential biological activity via pharmacology or toxicity. Indeed, where pharmacologically active metabolites are present, an understanding of the pharmacokinetic versus pharmacodynamic properties of the proposed therapy cannot be attained without such knowledge. However, in current drug-discovery and -development programs, the emphasis is on early administration to humans, in order to to justify further investment in developing the drug by determining likely efficacy as soon as possible. This has meant, in practice, that full characterization of the in vivo metabolic fate of drug candidates, in both animals and humans, based on gold-standard radiolabeled studies, has been pushed back in the development timetable and, in most cases, is performed during Phase II. There is currently strong reliance in the early stages of development on metabolic data provided by in vitro studies, usually performed in a limited range of human and animal cell types, most commonly hepatocytes. The recently published FDA guidance on MIST requires a paradigm shift, in so far as the emphasis is now moved firmly towards obtaining information on systemic metabolite exposure in humans as early as possible, to avoid costly delays in drug development [101].

\section{Background to the development of the guidance}

It is no surprise, based on the well-known importance of metabolites for safety and efficacy, that a number of regulatory guidelines have emphasized that (major) human metabolites should be covered in safety pharmacology studies (ICH guideline S7a [102]) and carcinogenicity protocol submissions [103].

\section{"The guidance is meant for small molecules only and does not yet apply to certain life- threatening indications, such as cancer..."}

The new MIST guidance from the FDA also deals, as the title suggests, with the role and implications of metabolites in safety studies [101]. The guidance provides protocols for those circumstances where the FDA deems it necessary to conduct special/additional safety studies for metabolites. The guidance is meant for small molecules only and does not yet apply to certain life-threatening indications, such as cancer, as it clearly states that "this guidance applies to small molecule nonbiologic drug products. This guidance does not apply to some cancer therapies where a risk-benefit assessment is considered."

The newer ICH S9 draft guidance on the nonclinical evaluation for anticancer pharmaceuticals clearly addresses this particular point [104]: "in some cases, metabolites have been identified in humans that have not been qualified in nonclinical studies. For those metabolites, a separate general toxicology evaluation might not be warranted for patients with late-stage or advanced cancer, as the human safety of the metabolite would have been assessed in Phase I clinical trials".

\section{Gerhard Gross ${ }^{\dagger}$ \& Ian Wilson}

†Author for correspondence

Department of Clinical

Pharmacology, Drug

Metabolism and

Pharmacokinetics,

AstraZeneca, Mereside,

Alderley Park, Macclesfield,

Cheshire SKIO 4TG, UK

Tel.: +44 I62 55। 5238

Fax: +44 1625516962

E-mail: gerhard.gross@

astrazeneca.com 
However, it seems likely that such a dispensation would be less likely to apply if the drugs were to be later proposed for early-stage disease, especially if long-term ( $>6$ month) administration were to be contemplated. Inherent to most preclinical toxicology programs is the assumption that the animal species used in these evaluations will serve as biological surrogates for humans. In situations in which drug-metabolite profiles reveal both quantitative and/or qualitative differences between the spectrum of metabolites formed in animal species and those formed in humans this particular assumption becomes questionable. The new guidance highlights two situations of special importance as follows:

- Humans may form a unique human-specific drug metabolite (or metabolites) not found in the animal species used in the preclinical evaluation;

- Humans may form a major metabolite only found in relatively low amounts in animals; the metabolite is disproportionate.

Under such circumstances, the animal species used for the preclinical toxicology evaluations cannot adequately assess the potential toxicity of the drug candidate and humans are exposed to a molecular species for which sufficient exposure is not attained in animals. The guideline defines these cases, suggests additional studies that should be performed and also gives a clear recommendation regarding the timing of these studies. It should be pointed out here that, in both circumstances, the FDA encourages early discussion, especially in complicating circumstances:"we encourage contacting the FDA early in drug development to discuss these issues."

The proposed guidance also gives a pragmatic definition for when metabolites should be considered under either Case 1 or 2, as described above: "human metabolites that can raise a safety concern are those formed at greater than $10 \%$ of parent drug systemic exposure at steady state. The choice of a level of greater than $10 \%$ for characterization of drug metabolites reflects consistency with FDA and Environmental Protection Agency guidance's."

The FDA allows, under certain circumstances, $\mathrm{C}_{\text {max }}$ to be included instead of the AUC at steady state $\left(\mathrm{AUC}_{\mathrm{ss}}\right)$ : "exposure should be at steady state unless there is some justification for a different measure of exposure. Comparison between human and animal exposure is generally based on the AUC, but sometimes it may be more appropriate to use $\mathrm{C}_{\max }$ " Besides consideration of systemic metabolites, the evaluation of metabolites in urine or feces is suggested in some cases: "adequacy of exposure to drug metabolites that are present at disproportionately lower levels in animals used in nonclinical studies should be considered on a case-by-case basis. Generally, systemic exposure is assessed by measuring the concentration of the parent drug at steady state, in serum or plasma. However, when measurements cannot be made in plasma of the test species for any reason, verification of adequate exposure can be made in other biological matrices such as urine, feces or bile”. It is also clearly pointed out that the $10 \%$ level might be supplemented in special cases by the need to evaluate the safety of metabolites present in excreta, as pointed out in footnote 10 from the guidance (Box I).

\section{"The US FDA encourages early discussion, especially in complicating circumstances..."}

The overall approach advocated by the FDA guidance for dealing with disproportionate metabolites is summarized in Figure I [101]. The guideline suggests two principal approaches to address disproportionate metabolites (these include human-specific metabolites and metabolites with a relatively low exposure in animals):

- "The first approach is to identify an animal species routinely used in toxicity studies that forms the metabolite at adequate exposure levels (equivalent to or greater than the human exposure) and then investigate the drug's toxicity in that species"

Box 1. Footnote 10 from the US FDA metabolites in safety testing guidance.

"Although the drug metabolite of toxicological concern usually is one circulating in plasma at greater than $10 \%$ of parent systemic exposure, other metabolites also can elicit safety concern. For example, a drug metabolite representing greater than $10 \%$ of urinary excretion relative to the bioavailable dose or a human fecal metabolite in cases where biliary elimination is the predominant route of excretion in humans, may reflect potential localized renal or bile duct toxicity, respectively. Further characterization in these instances should be addressed on a case-by-case basis with the review division." 
- "The second approach, if a relevant animal species that forms the metabolite cannot be identified, is to synthesize the drug metabolite and directly administer it to the animal for further safety evaluation"

Possible complications (e.g., metabolism of a metabolite administered to animals) mean that this might not reflect the clinical situation (e.g., due to species differences in metabolism or disposition) and may therefore complicate toxicological evaluation [1]. These complications are acknowledged in this guidance.

A comprehensive evaluation of all data is always recommended: "however, notwithstanding these possible complications, identification and evaluation of the potential toxicity of the drug metabolite is considered important to ensure clinical safety, and the decision to conduct direct safety testing of a metabolite should be based on a comprehensive evaluation of the data on the parent drug and any information available for the metabolite."

Four principal safety-study types are mentioned in the guidance document for assessing metabolite safety:

- Genotoxicity studies: if deemed necessary, a minimum screen for potential genotoxicity should be considered. In vitro studies to detect point mutations and chromosomal aberrations would be an example of a minimum screen;

- General toxicity studies: study duration should be based on available relevant information and performed in the species most likely to express potential toxicity (studies of the drug metabolite would not be required in two species). In general, 14-90 days would be considered appropriate study durations. It is recommended that the clinical route of administration that would be used for the parent is employed in such investigations on the metabolite;

- Reproductive toxicology studies: these would be appropriate when the parent drug may be administered in populations that include women who are pregnant or of child-bearing potential;

- Carcinogenicity testing, if appropriate: whether carcinogenicity testing is required for the parent drug depends on the intended indication for the parent drug. It is evident that if carcinogenicity testing is not necessary for approval of the parent compound (normally where the cumulative human lifetime exposure to the compound would not be expected to exceed 6 months or in life-threatening conditions), then such testing of the metabolite would not be required. "Carcinogenicity studies should be conducted on metabolites of drugs that are administered continuously for at least 6 months or that are used intermittently in the treatment of chronic or recurrent conditions when the carcinogenic potential of the metabolite cannot be adequately evaluated from carcinogenicity studies conducted with the parent drug" [101].

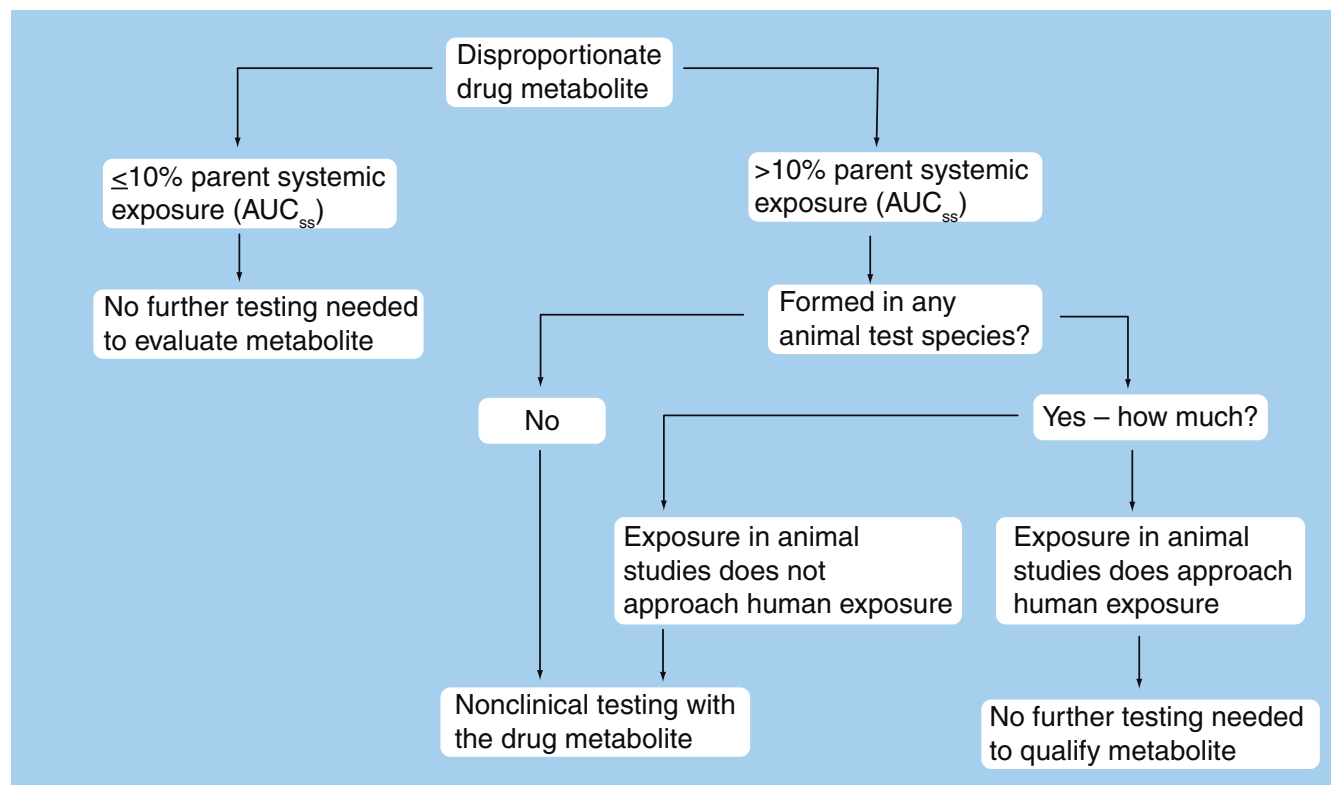

Figure 1. General US FDA approach to safety testing of metabolites. Modified from [101]. 
If there was evidence that the metabolite had caused lesions that might develop into neoplasia, but such lesions were not observed with the parent compound (i.e., they were metabolitespecific), then consideration should be given to carcinogenicity testing. Validated alternative short-term carcinogenicity assays could be considered for this assessment.

\section{Timing of metabolic studies}

The FDA guidance clearly indicates that studies to assess human metabolism should be conducted as early as possible: "we encourage the identification of differences in drug metabolism between animals used in nonclinical safety assessments and humans as early as possible during the drug-development process." It is also clearly stated in the guidance that all additional safety studies performed to investigate the safety of a metabolite should be finalized and reported to the FDA before the start of the Phase III clinical studies: "if toxicity studies of a drug metabolite are warranted, studies should be completed and study reports provided to the FDA before beginning large-scale clinical trials."

\section{Industrial strategies, challenges \& implications}

The principal response of the pharmaceutical industry to this new guidance must be to minimize the risk of discovering a unique or disproportionate metabolite in humans at a late stage of drug development by obtaining different sets of quality data regarding the human systemic metabolites as early as possible without employing excessive resources. Therefore, the key focus of many industrial responses to the guidance has been to employ the most meaningful models and advanced analytical techniques in order to maximize the readout at all stages of drug development. There are a number of publications outlining industrial approaches and experiences from different companies. A typical approach encompasses in vitro studies, in vivo studies in animals, clinical studies [2] and the application of techniques such as NMR spectroscopy [3]. Strategies for the detection of metabolites are likely to be based on maximizing the recovery of metabolism data from such studies using sensitive, informationrich analytical technologies (e.g., LC-MS and NMR spectroscopy), perhaps combined with advanced pattern-recognition methods to tease out metabolites that could be missed by conventional methods [4]. Mass filtering techniques coupled with computer-assisted structure-elucidation software, with the MS response from radioactive in vitro metabolic profiles used to enable quantification, is an elegant approach to maximizing the overall readout of in vivo data to estimate the amounts of metabolites present in human plasma samples [5]. As we have shown elsewhere, the presence of particular atoms as substituents in molecules, such as the halogens fluorine, bromine, iodine (and to a lesser extent chlorine) or the elements phosphorous and sulphur, can enable quantitative and specific metabolite profiles to be obtained without need for radiolabels [6]. Similarly, the use of cocktails of drug and stable isotope-labeled versions to produce mixtures with a distinctive MS 'signature' facilitates their detection and requires less preclinical work than radiolabeled studies in humans $[4,7]$. However, whilst these isotope-based MS approaches enable the detection of drug-derived material, they do not allow the quantification of the metabolites in the absence of standards. Providing that samples from the pivotal preclinical animal toxicology studies are retained, it should always be possible to retrospectively search for any unexpected metabolites detected in humans. However, such approaches clearly require good coordination between groups (clinical, preclinical and toxicological) and careful collection and appropriate storage of samples.

"As every approach has some intrinsic
difficulties, the data provided by each model
therefore have some limitations..."
Another potential approach for maximiz-
ing the readout from early studies concerning
human systemic exposure of metabolites, in
addition to those outlined above, is the appli-
cation of accelerator MS (AMS); some suc-
cessful applications of this method have been
published [8,9]. While in a conventional human
ADME study the amount of administered
${ }^{4} \mathrm{C}-$ labeled drug is typically approximately 100
$\mu \mathrm{Ci}(3-4 \mathrm{MBq}$ ), in AMS the radioactive dose
can be reduced by an order of magnitude (i.e.,
$3.7-7.4 \mathrm{kBq}$ ). Since the human body on average
contains approximately $3.7 \mathrm{kBq}$ of naturally
occurring ${ }^{14} \mathrm{C}$, this type of study can be con-
idered effectively to be a nonradioactive study
and the dosimetry calculations required for a
conventional human radiolabeled metabolism

Another potential approach for maximizing the readout from early studies concerning human systemic exposure of metabolites, in addition to those outlined above, is the application of accelerator MS (AMS); some successful applications of this method have been published [8,9]. While in a conventional human ADME study the amount of administered ${ }^{14} \mathrm{C}$-labeled drug is typically approximately 100 $\mu \mathrm{Ci}(3-4 \mathrm{MBq})$, in AMS the radioactive dose can be reduced by an order of magnitude (i.e., $3.7-7.4 \mathrm{kBq}$ ). Since the human body on average contains approximately $3.7 \mathrm{kBq}$ of naturally occurring ${ }^{14} \mathrm{C}$, this type of study can be considered effectively to be a nonradioactive study conventional human radiolabeled metabolism 
studies may be unnecessary. It is not currently possible to directly obtain metabolite profiles using AMS, and these can only be obtained by fractionating the samples (e.g., plasma, urine and fecal extracts) by HPLC with subsequent determination of the radioactivity in each fraction, and then constructing a metabolite profile from the data [10]. AMS thus provides a strategic option for an early Phase I clinical study to obtain comprehensive information regarding the systemic metabolite pattern in humans early in development. However, such an approach has drawbacks; the final clinical dose is typically not known at this early stage of development, which might cause complications at later stages, as systemic metabolite exposure might vary with dose. Also to be mentioned is that the experimental procedures involved in this approach are usually very resourceintensive and costly. It is possible, however, that through good strategic planning and by minimizing the number of samples to be investigated, these aspects can, to some extent, be counterbalanced.
As every approach has some intrinsic difficulties, the data provided by each model therefore have some limitations, as TABLE I indicates.

It is evident that each approach has its limitations and complications. Recent publications discuss some examples in depth $[2,3,5]$. A case study from Elli Lilly analyzed data on 17 molecules, where in vitro metabolism data were compared with the results from the human radiolabeled studies, with respect to the predictive power of this approach [11]. Approximately $41 \%$ of the cases resulted in an accurate prediction, in $35 \%$ of the cases in vitro data underpredicted the circulating metabolites and in $24 \%$ of the cases metabolites were overpredicted. This relatively poor degree of prediction is partly due to the fact that a stationary in vitro system was compared with a dynamic in vivo system; a trend was noted that the more complex the metabolism, the poorer the in vitro data prediction of the circulating metabolites. In vitro data seemed also to be less predictive for noncytochrome P450 (CYP450)-mediated metabolism and $N$-glucuronidations [13].

\section{Table 1. Methods for metabolite detection and identification.}

\begin{tabular}{|c|c|c|c|c|c|}
\hline Sample & Model & Readout & Techniques & Limitations & Ref. \\
\hline $\begin{array}{l}\text { In vitro metabolism } \\
\text { with nonradiolabeled } \\
\text { material }\end{array}$ & $\begin{array}{l}\text { Liver models, such as } \\
\text { hepatocytes and } \\
\text { liver microsomes }\end{array}$ & $\begin{array}{l}\text { Qualitative } \\
\text { detection of } \\
\text { metabolites with } \\
\text { structural } \\
\text { identification }\end{array}$ & Mostly LC-MS/MS & $\begin{array}{l}\text { Provides limited, essentially qualitative, } \\
\text { information on metabolism. If a } \\
\text { metabolite gives insufficient MS } \\
\text { response it may be missed. Model is } \\
\text { generally better at predicting clearance } \\
\text { pathways than circulating metabolites. } \\
\text { The prediction of systemic exposure is } \\
\text { not always reliable }\end{array}$ & \\
\hline $\begin{array}{l}\text { In vitro metabolism } \\
\text { with radiolabeled } \\
\text { compound }\end{array}$ & $\begin{array}{l}\text { Liver models, such as } \\
\text { hepatocytes and } \\
\text { liver microsomes }\end{array}$ & $\begin{array}{l}\text { Quantitative } \\
\text { proportions of } \\
\text { metabolites with } \\
\text { structural } \\
\text { identification }\end{array}$ & $\begin{array}{l}\text { Radioactivity } \\
\text { detection coupled } \\
\text { with LC-MS/MS } \\
\text { or NMR }\end{array}$ & $\begin{array}{l}\text { Model is insufficient for extrahepatic } \\
\text { metabolism. It is generally better at } \\
\text { predicting clearance pathways than } \\
\text { circulating metabolites. Metabolite } \\
\text { pattern varies with incubation time and } \\
\text { concentration especially for } \\
\text { high-clearance drugs }\end{array}$ & {$[12,13]$} \\
\hline $\begin{array}{l}\text { Single/multiple dose } \\
\text { studies in human }\end{array}$ & Human & $\begin{array}{l}\text { Systemic exposure } \\
\text { of metabolites }\end{array}$ & LC-MS/MS & $\begin{array}{l}\text { Reliable quantification is only possible } \\
\text { with reference standard, the use of UV } \\
\text { or MS response factors from } \\
\text { radiolabeled in vitro study possible }\end{array}$ & \\
\hline $\begin{array}{l}\text { Human radiolabeled } \\
\text { study }\end{array}$ & Human & $\begin{array}{l}\text { Systemic exposure } \\
\text { of metabolites, } \\
\text { metabolite pattern } \\
\text { in urine and feces, } \\
\text { mass balance and } \\
\text { excretion pathways }\end{array}$ & $\begin{array}{l}\text { Radioactivity } \\
\text { detection coupled } \\
\text { with LC-MS/MS, } \\
\text { NMR }\end{array}$ & $\begin{array}{l}\text { The gold standard. Full readout } \\
\text { is possible, but can only be undertaken } \\
\text { after significant preclinical studies } \\
\text { and after regulatory approval has } \\
\text { been obtained }\end{array}$ & \\
\hline
\end{tabular}




\section{Causes of variation in the systemic exposure of metabolites}

There are a number of other challenges and variables that also contribute towards the complexity of the metabolite issue and are significant for drug development with respect to the FDA guidance. Common parameters that can have significant impact on the systemic exposure of a metabolite include variables such as age [14] sex and type of population considered (e.g., Caucasian, Asian or Afro-American) [15], all of which could alter the systemic exposure of a particular metabolite and therefore impact on whether a metabolite would be considered 'MIST' relevant or not. If certain metabolic steps are subject to genetic polymorphism, there may be significant differences in the overall formation rate of a particular metabolite, thus resulting in different systemic exposure of this metabolite between poor and extensive metabolizers. Some metabolites show much lower volumes of distribution compared with the parent drug, which might cause a much higher systemic exposure, despite the fact that the overall body burden is quite low. An example given in a recent review illustrates this: a metabolite of the compound CP-122721 represented more than 50\% of systemic exposure but accounted for only $1.4 \%$ of the total dose in the excreta [16]. Similarly, enzyme or transporter inhibition [17] as well as enzyme/transporter induction [18] can trigger significant changes in systemic exposure. A striking example of changes in systemic exposure has been shown in rats, where the induction of mrp3 by phenobarbital caused significant higher systemic exposure of acetaminophen-glucuronide [19].

\section{Relevant species differences in metabolism}

Human-specific or disproportionate metabolites are mostly the result of species differences in drug metabolism or distribution between tissues and blood [16,20-22]. These differences in metabolism can either be qualitative or quantitative. In some cases, even the inhibition is found to be species specific, such as in the case of CYP2D inhibition by quinidine, where quinidine inhibits CYP2D6 in humans but not in mice [21]. Major differences in metabolism are found in the CYP2C family [22], with NAT2 [23], the aldehyde oxidase [24] and $N$-glucuronidation of tertiary amine drugs, where UGT1A4 plays a key role [11,16,25].

\section{New models for humans}

There is no doubt that the development of better preclinical models for humans would greatly help to derisk issues surrounding human-specific metabolites in early development. Newer in vivo models, such as the humanized mouse, have shown promising results where a urokinase-type plasminogen activator $+/+/$ severe combined immunodeficient transgenic mouse line had more than $80 \%$ of its liver replaced with human hepatocytes [26]. A study examining the hydroxylation of $S$-warfarin showed that the 7-hydroxylase activity of liver microsomes towards $S$-warfarin was tenfold higher in microsomes from the chimeric mouse. Systemic exposure of this metabolite in the chimeric mice was sevenfold higher, in agreement with in vitro findings [27]. A recent investigation into species differences in aldehyde oxidase used $N(1)$-methylnicotinamide as a substrate; $N(1)$-methylnicotinamide is metabolized to $N(1)$-methyl-2-pyridine-5-carboxamide (2-PY) and $N(1)$-methyl-4-pyridone-5-carboxamide (4-PY) through aldehyde oxidase. In vitro chimeric mouse activity in liver cytosol was fourfold higher than in control mice. Furthermore, the metabolism was also altered. In the control mice, 2-PY and 4-PY were found, while in chimeric mice 2-PY was the major product, as in humans. The ratio of 2-PY/4-PY in the urine of chimeric mice was closer to that of humans [28]. Humanization of excretion pathways was also achieved with this model. Cefmetazole excretion in urine and feces was found to be 81 and 5.9\% of the dose in chimeric mouse compared with 23.7 and $59.4 \%$ in control mice. Since cefmetazole is excreted mainly via urine in humans, this seems to indicate that excretion can also be humanized [29].

\section{"There is no doubt that the development of better preclinical models for humans would greatly help to derisk issues surrounding human-specific metabolites in early development. Newer in vivo models, such as the humanized mouse, have shown promising results..."}

In conclusion, providing that metabolism is hepatic, the application of this chimeric mouse model is likely to provide insight, although in a qualitative to semiquantitative manner, into the likely human metabolism than many of the conventional in vitro models currently applied.

\section{MIST guidance: scientific discussion}

The most discussed aspect of the FDA guidance is the use of a threshold of $10 \%$ of parent $\mathrm{AUC}_{\text {ss }}$ as the trigger [101]. The guidance refers to this 
particular threshold as: "the choice of a level of greater than $10 \%$ for characterization of drug metabolites reflects consistency with FDA and Environmental Protection Agency guidances."

The MIST draft guidance was discussed in detail in a recent review [30]. This review summarizes the opinions gathered at a recent drug evaluation forum in Japan with representatives from the Japanese Pharmaceutical and Medical Devices Evaluation Center, as well as people from academia and industry. This article also provides a comprehensive summary of past discussions [31-34,105]. The main recommendation was that there should be a case-by-case decision regarding the threshold rather than a general rule (e.g., 10\% of AUC). It should be pointed out that the major part of the scientific discussion about the MIST guideline is well documented in the current literature. In particular, there were discussions concerning the use of percentagebased versus abundance criteria with respect to exposure [33], consideration of mechanisms of toxicity and duration of treatment [16], as well as the practical difficulties of investigating metabolite safety by dosing a metabolite as this might significantly alter overall disposition compared with the metabolite formed following administration of parent compound [1]. Part of these considerations and the underlying difficulties are clearly acknowledged in the MIST guidance.

As we indicated earlier, the FDA guidance explicitly states that $10 \%$ of the AUC of the parent compound at steady state is the central criteria for assessing whether or not a metabolite qualifies as being subject for further safety investigations. The majority of human ADME studies, considered to be the gold standard for assessing metabolism in humans, are typically performed as single-dose studies for ethical, practical and legal reasons. It is possible to estimate the AUC of a particular metabolite at steady state of parent compound through the extrapolation/superposition from a single-dose study or specifically determine metabolite exposure from clinical multipledose studies [35,36]; all extrapolations require that parent compound and metabolite(s) follow linear processes. Therefore, previous findings from animal studies concerning systemic exposure and kinetics of metabolites concerning indications of potential drug/metabolite accumulation must be integrated into the overall strategy. Information on the pharmacokinetic parameters of the major metabolites from the in vivo studies in the preclinical animal species are therefore very helpful in assuring adequate planning of the hADME study.
This implies that all single-dose radiolabeled studies should be designed to allow extrapolation of the data for circulating metabolites to steady state of parent drug. Where a human metabolite shows disproportionate accumulation to concentrations likely in animals in safety studies further investigations should be made, using specific analytical methods, in both the preclinical species and humans.

Another potential option is to perform the hADME study as part of a multiple-dose study and dose the single radioactive dose when the subjects have achieved steady state for the parent compound [37]. It should be stated here that such an approach is currently not the industry standard for the reasons discussed previously.

\section{"All single-dose radiolabeled studies should be designed to allow extrapolation of the data for circulating metabolites to steady state. Where $a$ human metabolite shows disproportionate accumulation to concentrations likely in animals in safety studies further investigations should be made..."}

There are cases where systemic concentrations/exposures of parent/metabolite do not reflect toxicity at the target site. If clear evidence exists for such, we recommend modification of the overall approach accordingly. When the parent compound forms a very low percentage of total (metabolites and parent) $\mathrm{AUC}_{\mathrm{ss}}$, the $10 \%$ criteria becomes very challenging to apply; we recommend a discussion with the FDA in such cases.

Drugs with very pronounced metabolisms, encompassing several generations of metabolites (e.g., primary or secondary), will often show very pronounced differences in metabolism across species and doses [38], as the metabolite pattern in general includes a mixture of numerous minor primary, secondary and tertiary metabolites. In such cases, it is arguable that, if the primary metabolic pathways across species are the same, this renders the animal species acceptable for the assessment of human safety. Such a statement implies that second- and further-generation metabolites are not considered a concern for safety. This statement partly follows the line of argument used for Phase II metabolites given in the guidance: "Phase II conjugation reactions generally render a compound more water soluble and pharmacologically inactive, thereby eliminating the need for further evaluation." 
The FDA guidance acknowledges that, in principle, Phase II metabolites are generally of no concern. However, it does specifically mention acyl-glucuronides as being of potential concern: "however, if the conjugate forms a toxic compound such as acyl glucuronide, additional safety assessment may be needed". It is therefore our opinion that it is necessary for any potentially acyl-glucuronide-related toxicological findings to be followed up and that the systemic exposure of these metabolites is determined as early as possible in respect of the critical opinion of the FDA in this matter. It is also our opinion that the reactivity and overall body burden of the conjugate should be considered.

\section{Summary \& recent developments}

The new FDA MIST guidance provides clear criteria under which circumstances a metabolite should be considered as 'safety relevant' and, thus, undergo further toxicity assessment.

The FDA guideline on MIST provides considered practical guidance regarding what needs to be done to address potential safety concerns that may be posed by drug metabolites going forward. This guidance has already had a significant impact on the pharmaceutical industry as early information on the systemic metabolite pattern in humans becomes key in order not to lose precious development time due to a disproportionate metabolite issue. The industry has established a number of strategies and approaches to address this topic, although these still contain some inherent uncertainties and challenges. However, it is clear that the science behind this document is still rapidly evolving and there is clear flexibility within the FDA, with respect to the development of individual compounds, to ensure that the delivery of innovative therapies to patients is not compromised.
Indeed, not only is the science behind the regulatory approach to metabolites evolving but so is that of the regulatory bodies themselves. Newer guidance has very recently been published on this topic (ICH M3(R2) that is particularly relevant to this topic [39]. This guidance, entitled 'Nonclinical safety studies for the conduct of Human Clinical Trials and marketing authorization for Pharmaceuticals' also discusses metabolite safety issues [39]. The major change compared with the FDA guidance is that this guidance explicitly states that a human metabolite is only safety relevant if its exposure is greater $10 \%$ of total drug-related exposure, compared with $10 \%$ of parent $\mathrm{AUC}_{\mathrm{ss}}$ in the FDA document, and at significantly higher levels in humans than observed in animal toxicity studies. For low-dose compounds (i.e., $<10 \mathrm{mg}$ ), it also states that even higher systemic levels are necessary to trigger additional metabolite safety testing. Metabolites that cause special concern, such as unique human metabolites, are proposed to be considered on a case-by-case basis before subjecting them to any nonclinical characterization. Phase II metabolites are also mentioned as not being of toxicological concern.

Clearly, the subject of metabolites and their role in safety, has triggered an ongoing debate, as further evidenced by recent publications [40], and new refinements and adaptations can be expected as a result of the rapidly moving science and increased understanding of this area.

\section{Financial \& competing interests disclosure}

The authors have no relevant affliations or financial involvement with any organization or entity with a financial interest in or financial conflict with the subject matter or materials discussed in the manuscript. This includes employment, consultancies, honoraria, stock ownership or options, expert testimony, grants or patents received or pending, or royalties.

No writing assistance was utilized in the production of this manuscript.

\section{Bibliography}

Papers of special note have been highlighted as:

- of interest

"- of considerable interest

1 Prueksaritanont T, Lin JH, Baillie TA. Complicating factors in safety testing of drug metabolites. kinetic differences between generated and preformed metabolites. Toxicol. Appl. Pharmacol. 217, 143-152 (2006).

2 Leclercq L, Cuyckens F, Mannens GS et al. Which human metabolites have we MIST? Retrospective analysis, practical aspects and perspectives for metabolite identification and quantification in pharmaceutical development. Chem. Res. Toxicol. 22, 280-293 (2009).

- Essential reading to understand the difficulties of predicting the systemic exposure of metabolites.

3 Espina R, Yu L, Wang J, Tong Z, Vashishtha $S$ et al. Nuclear magnetic resonance spectroscopy as a quantitative tool to determine the concentrations of biologically produced metabolites: implications in metabolites in safety testing. Chem. Res. Toxicol. 22, 299-310 (2009).

4 Athersuch TJ, Nicholson JK, Wilson ID. Isotopic enrichment enhancement in metabonomic analysis of UPLC-MS data sets. J. Label. Compd Radiopharm. 50 303-307 (2007).

5 Baillie TA. Approaches to the assessment of stable and chemically reactive drug metabolites in early clinical trials. Chem. Res. Toxicol. 22, 263-266 (2009). 
6 Smith CJ, Shillingford S, Edge AM et al. Quantification of the in vitro and in vivo metabolic fates of 2-, 3- and 4-bromobenzoic acids using high temperature LC coupled to ICP-MS and linear ion trap MS. Chromatographia 67N, 673-678 (2008).

- Useful demonstration of alternative methods to radioactivity for the quantification of metabolites.

7 Zhu P, Tong W, Alton K et al. An accuratemass-based spectral averaging isotopepattern-filtering algorithm for extraction of drug metabolites possessing a distinct isotope pattern from LC-MS data. Anal. Chem. 81, 5910-5917 (2009).

- Interesting approach to metabolite detection by MS.

8 Lappin G, Stevens L. Biomedical accelerator mass spectrometry: recent applications in metabolism and pharmacokinetics. Expert Opin. Drug Metab. Toxicol. 4, 1021-1033 (2008).

9 Skerjanec A, Berenson J, Hsu C et al. The pharmacokinetics and pharmacodynamics of zoledronic acid in cancer patients with varying degrees of renal function. J. Clin. Pharmacol. 43,154-162 (2003)

10 Prakash C, Shaffer CL, Tremaine LM et al. Application of liquid chromatography-accelerator mass spectrometry (LC-AMS) to evaluate the metabolic profiles of a drug candidate in human urine and plasma. Drug Metab Lett. 1(3), 226-231 (2007).

11 Anderson S, Luffer-Atlas D, Knadler MP. Predicting circulating human metabolites. How good are we? Chem. Res. Toxicol. 22, 243-256 (2009).

12 Dalvie D, Obach RS, Kang P et al. Assessment of three human in vitro systems in the generation of major human excretory and circulating metabolites.Chem. Res. Toxicol. 22. 357-368 (2009).

13 Luffer-Atlas D. Unique/major human metabolites: why, how and when to test for safety in animals. Drug Metab. Rev. 40, 447-463 (2008).

14 Hilmer SN. ADME-tox issues for the elderly. Expert Opin. Drug Met. Toxicol. 4, 1321-1331 (2008).

15 Johnson DE, Park BK, Smith DA. Ethnic variation in drug response: implications for the developments and regulation of drugs. Curr. Opin. Drug Disc. Devel. 11, 29-31 (2008).

16 Smith DA, Obach RS. Metabolites in safety testing (MIST): considerations of mechanisms of toxicity with dose, abundance and duration of treatment. Chem. Res.Toxicol. 22, 267-279 (2009).

- Key paper in the overall scientific discussion of metabolites and safety.

17 Nassar AEF, Talaat RE, Tokane H. Drug interactions: concerns and current approaches. IDrugs 10, 47-52 (2007).

18 Urquhart BL, Tirona RG, Kim RB. Nuclear receptors and the regulation of drug-metabolizing enzymes and drug transporters. Implications for interindividual variability in response to drugs. J. Clin. Pharmacol. 47, 566-578 (2007).

19 Klaassen CL, Slitt AE. Regulation of hepatic transporters by xenobiotic receptors. Curr. Drug Met. 6(4), 309-328 (2005).

20 Martignoni M, Groothuis GM, de Kanter R. Species differences between mouse, rat, dog, monkey and human CYP-mediated drug metabolism, inhibition and induction. Expert Opin. Drug Metab. Toxicol. 2, 875-894 (2006).

21 Bogaards JJ, Bertrand M, Jackson P et al. Determining the best animal model for human cytochrome $\mathrm{P} 450$ activities: a comparison of mouse, rat, rabbit, dog, micropig, monkey and man. Xenobiotica. 30, 1131-1152 (2000).

-. Key review regarding species differences in metabolism.

22 Graham MJ, Bell AR, Crewe HK et al. mRNA and protein expression of dog liver cytochromes $\mathrm{P} 450$ in relation to the metabolism of human CYP2C substrates. Xenobiotica. 33, 225-237 (2003).

23 Gao W, Jeffrey S, Johnston JS, Miller DD et al. Interspecies differences in pharmacokinetics and metabolism of $S$-3-(4-acetylamino-phenoxy)-2hydroxy-2-methyl- $N$-(4-nitro-3trifluoromethylphenyl)-propionamide: the role of $N$-acetyltransferase. Drug Metab. Dispos. 34, 254-260 (2006).

24 Sahi J, Khan KK, Black CB. Aldehyde oxidase activity and inhibition in hepatocytes and cytosolic fractions from mouse, rat, monkey and human. Drug Metab. Lett. 2, 176-183 (2008).

25 Lee S-H, Chiu S-HL, Huskey S-EW. Species differences in $N$-glucuronidation. Drug Metab. Dispos. 26, 838-847 (1998).

26 Katoh M, Yokoi T. Application of chimeric mice with humanized liver for predictive ADME. Drug Metab. Rev. 39, 145-157 (2007).
27 Inoue T, Nitta K, Sugihara K et al. CYP2C9catalyzed metabolism of $S$-warfarin to 7-hydroxywarfarin in vivo and in vitro in chimeric mice with humanized liver. Drug Metab. Dispos. 36. 2429-2433 (2008).

28 Kitamura S, Nitta K, Tayama Y et al. Aldehyde oxidase-catalyzed metabolism of N1-methylnicotinamide in vivo and in vitro in chimeric mice with humanized liver. Drug Metab. Dispos. 36. 1202-1205 (2008).

29 Okumura H, Katoh M, Sawada T et al. Humanization of excretory pathway in chimeric mice with humanized liver. Toxicol. Sci. 97, 533-538 (2007).

30 Naito S, Furuta S, Yoshida T et al. Current opinion: safety evaluation of drug metabolites in development of pharmaceuticals. J. Toxicol. Sci. 32, 329-341 (2007).

31 Baillie TA, Cayen MN, Fouda H et al. Drug metabolites in safety testing. PhRMA position paper. Toxicol. Appl. Pcol. 182, 188-196 (2002).

32 Baillie TA. MIST-Reply (Letter to the Editor) Toxicol. Appl. 190, 91-92 (2003).

33 Smith DA, Obach RS. Seeing through the mist: abundance versus percentage. Commentary on metabolites in safety testing. Drug Metab. Dispos. 33, 1409-1417 (2005).

34 Davis-Bruno KL, Atrakchi A. A regulatory perspective on issues and approaches in characterizing human metabolites. Chem. Res. Toxicol. 19, 1561-1563 (2006).

35 Handbook of Drug Metabolism (2nd Edition). Pearson PG, Wienkers LC (Eds). Informa Healthcare, London, UK, 37-38 (2008).

36 Lane EA, Levy RH. Prediction of steady state behavior of metabolite from dosing of parent drug. J. Pharm. Sci. 69, 610-612 (1980).

37 Jost LM, Gschwind HP, Jalava T et al. Metabolism and disposition of vatalanib (PTK787/ZK-22584) in cancer patients. Drug Met. Disp. 34, 1817-1828 (2006).

38 Zollinger M, Waldmeier F, Hartmann S et al. Pimecrolimus: absorption, distribution, metabolism and excretion in healthy volunteers after a single oral dose and supplementary investigations in vitro. Drug Metab. Dispos. 34, 765-774 (2006).

39 ICH Topic M3 (R2). Non-clinical safety studies for the conduct of human clinical trials and marketing authorization for pharmaceuticals. CPMP/ICH/286/95. June 2009.

40 Atrakchi AH. Interpretation and considerations on the safety evaluation of human drug metabolites. Chem. Res. Toxicol. 22, 1217-1220 (2009). 


\section{COMMENTARY | Gross \& Wilson}

\section{- Websites}

101 US FDA. Guidance for Industry: Safety Testing of Drug Metabolites. Center for Drug Evaluation and Research, Rockville, MD, USA (2008).

-. Essential reading in order to understand the requirements of the US FDA.

102 ICH S7A safety pharmacology studies for human pharmaceuticals www.fda.gov/cder/guidance/4461fnl.pdf
103 Guidance for industry carcinogenicity study protocol submissions www.fda.gov/cder/guidance/4804fnl.pdf

104 ICH S9 nonclinical evaluation for anticancer pharmaceuticals, draft guidance www.fda.gov/cder/guidance/8681dft.pdf
105 Shiew-Mei Huang. Making a decision on drug metabolism data - go or no go. Presented at: AAPS-PPDM round table. San Diego, CA, USA, 12 November 2007 www.fda.gov/cder/drug/druginteractions/ presents/2007-2012-2011_huang_20_min_ final_bw.pdf 